\title{
Inteiros algébricos e a questão da comensurabilidade entre o lado e as diagonais de um polígono regular
}

\author{
Ronald S. de M. Pinto Diego Alves
}

\section{Resumo}

Neste artigo, apresentamos um estudo sobre a comensurabilidade entre as diagonais e o lado de polígonos regulares. Começando por fornecer uma fórmula para a razão entre os comprimentos da $k$-ésima diagonal mais curta e do lado de um polígono regular de $n$ lados, iremos reduzir o estudo da questão da incomensurabilidade entre a $k$-ésima diagonal mais curta e o lado de um polígono regular de $n$ lados à decisão pela racionalidade ou irracionalidade dessa razão. Para tal, utilizaremos os números inteiros algébricos.

Palavras-chave: Números irracionais; inteiros algébricos; segmentos incomensuráveis e polígonos regulares.

\section{Abstract}

In this article, we present a study on the commensurability between the diagonals and the side of regular polygons. Beginning by providing a formula for the ratio between the lengths of the shortest k-th diagonal and the side of a regular n-sided polygon, we will reduce the study of the question of the incommensurability between the $\mathrm{k}$-th shortest diagonal and the side of a regular polygon of $\mathrm{n}$ sides to the decision of the rationality or irrationality of this reason. For this, we will use the algebraic integers.

Keywords: Irrational numbers; algebraic integers; incommensurable segments; regular polygons.

\section{Introdução}

Sabe-se da existência de segmentos incomensuráveis desde a Antiguidade, ver [5]. Não se conhece ao certo, no entanto, o primeiro exemplo de dois segmentos incomensuráveis. Acredita-se, contudo, que os incomensuráveis tenham surgido no estudo da diagonal do quadrado (que nos leva ao número $\sqrt{2}$ ) ou das diagonais do pentágono regular (que nos leva ao número de ouro $\phi$, ver [3]), que constituem o famoso pentagrama. É natural analisarmos a questão da comensurabilidade entre as diagonais e o lado de outros polígonos regulares. Ao ao contrário, porém, do quadrado e do pentágono regular, polígonos com mais de 5 lados possuem diagonais não congruentes. Devemos falar então da diagonal mais curta, segunda diagonal mais curta e assim sucessivamente. O Portal Atractor, ver [1], apresenta o estudo da comensurabilidade entre o lado e diagonal mais curta e o 
lado e a segunda diagonal mais curta de qualquer polígono regular. Mas não faz menção a outras diagonais, isto é, a partir da terceira diagonal mais curta. O que ocorre, por exemplo, com a medida do lado e da diagonal do dodecágono regular? Trata-se de segmentos comensuráveis ou incomensuráveis? No presente artigo, utilizando os inteiros algébricos, vamos examinar a questão da comensurabilidade entre o lado e outras diagonais de qualquer polígono regular. Para isso, iremos obter uma expressão para a razão da medida de uma diagonal e a medida do lado de um polígono regular, e, a partir daí, concluir que tal razão é um inteiro algébrico (real). Como os inteiros algébricos (reais) são números inteiros ou números irracionais, o estudo da comensurabilidade entre a medida de uma diagonal e a medida do lado de um polígono regular reduz-se a decidir se tal razão é, ou não, um número inteiro.

\section{A razão entre a diagonal e o lado de um polígono regular}

Vamos começar obtendo uma fórmula para a razão entre a medida da $k$-ésima diagonal mais curta e a medida do lado de um polígono regular de $n$ lados, onde $n$ e $k$ são inteiros não negativos.

A primeira questão com o qual deparamos consiste em saber para que valores de $k$ faz sentido falar da $k$-ésima diagonal mais curta de um polígono regular de $n$ lados, onde $n \geq 4$ e $k$ são números inteiros não negativos (em alguns momentos será útil interpretarmos o lado do polígono regular como a 0-ésima diagonal mais curta). Após respondermos esse ponto, iremos fornecer uma fórmula para a razão entre os comprimentos da $k$-ésima diagonal mais curta e do lado de um polígono regular de $n$ lados, onde $n \geq 4$ e $k$ são inteiros positivos, e expressar tal fórmula por meio de um polinômio em $2 \cos \frac{\pi}{n}$.

Dado um polígono regular regular de $n$ lados e vértices $V_{1}, V_{2}, \ldots, V_{n}$, se $n$ for par, então para cada $i=1, \ldots, \frac{n}{2}$ a reta $V_{i} V_{i+\frac{n}{2}}$ é um eixo de simetria do polígono. Assim, das $n-3$ diagonais que passam por $V_{i}$, temos uma (e apenas uma) diagonal maior, que é a $V_{i} V_{i+\frac{n}{2}}$, e as restantes $n-4$ diagonais são iguais duas a duas (veja o polígono regular de doze lados na Figura (1)). Há então $\frac{n-4}{2}+1$ diagonais diferentes. Por isso, só faz sentido falar da $k$-ésima diagonal mais curta quando $k \leq \frac{n-4}{2}+1$. Ou seja, quando

$$
n \geq 2 k+2 .
$$

Por outro lado se $n$ for ímpar, a reta $V_{i} M_{i}$, onde $M_{i}$ é o ponto médio do lado oposto a $V_{i}$, é um eixo de simetria do polígono. Dessa forma, as $n-3$ diagonais que passam por $V_{i}$ são iguais duas a duas (veja o polígono regular de onze lados na Figura (1)). Neste caso existem $\frac{n-3}{2}$ diagonais diferentes, de forma que só faz sentigo falar da $k$-ésima diagonal mais curta quando $k \leq \frac{n-3}{2}$, ou seja, quando

$$
n \geq 2 k+3 .
$$

De (1) e (2) resulta que só faz sentido falarmos da $k$-ésima diagonal mais curta de um polígono regular de $n$ lados quando $n \geq 2 k+2$. Assim, no que segue, tomaremos os inteiros $k$ e $n$ que satisfaçam a Desigualdade (1).

Dessa forma, para a segunda diagonal mais curta o polígono regular deve ter ao menos $2 \cdot 2+2=6$ lados; para a terceira diagonal mais curta o polígono regular deve ter ao menos $2 \cdot 3+2=8$ lados e 

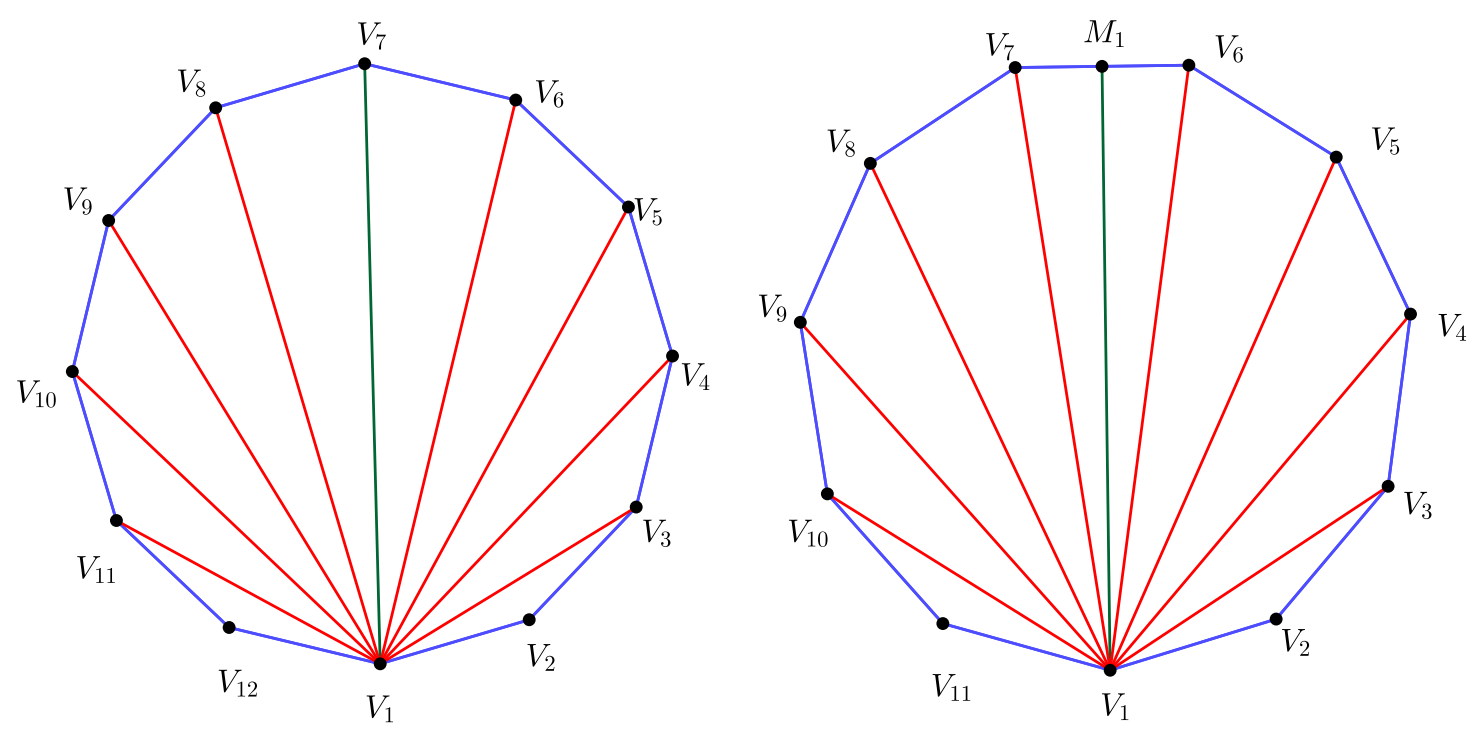

Figura 1: Só faz sentido falarmos da $5^{\text {a }}$ diagonal mais curta para polígonos com ao menos doze lados.

assim sucessivamente. Por exemplo, para tratarmos da $5^{\text {a }}$ diagonal mais curta, veja a Figura (1), o polígono deve ter ao menos $2 \cdot 5+2=12$ lados.

Dado um polígono regular de $n$ lados, vamos denotar por $\lambda_{k, n}$ a razão entre a medida do comprimento da $k$-ésima diagonal mais curta e a medida do comprimento do seu lado, onde $n \geq 2 k+2$. Por exemplo, a medida da quarta diagonal mais curta do dodecágono regular de lado medindo 1 , veja a Figura (2), é denotada por $\lambda_{4,12}$.

Com o objetivo de encontrarmos a razão entre a $k$-ésima diagonal mais curta e o lado de um polígono regular de $n$ lados utilizaremos números complexos. Para isso, seja $P_{n}$ o polígono regular de $n$ lados inscrito na circunferência unitária $\{z \in \mathbb{C} ;|z|=1\}$, com um vértice no ponto $z=1$.

Sabemos que, no plano complexo, as raízes n-ésimas da unidade (isto é, as raízes da equação $z^{n}=1$ ) são os vértices do polígono regular $P_{n}$ de $n$ lados, inscrito na circunferência unitária $\{z \in \mathbb{C} ;|z|=1\}$, com um vértice no ponto $z=1$, ver [2]. A Figura (3) representa o polígono $P_{7}$.

Como todos os polígonos regulares de $n$ lados são semelhantes, o nosso problema reduz-se a achar a razão entre a medida da $k$-ésima diagonal mais curta e a medida do lado do polígono regular $P_{n}$. Como mostra a referência [2], as n-ésimas raízes da unidade são dadas por

$$
\xi_{k}=\cos \left(\frac{2 \pi}{n} k\right)+i \operatorname{sen}\left(\frac{2 \pi}{n} k\right), k=0,1,2, \ldots, n-1 .
$$

Vamos denotar por $|z|$ e $\bar{z}$, respectivamente, o módulo e o conjugado do número complexo $z$. Lembre-se que $|z|^{2}=z \cdot \bar{z}$ e que $z+\bar{z}=2 \mathcal{R}(z)$, onde $\mathcal{R}(z)$ denota a parte real de $z$. Com essas observações, estamos em condições de provar o lema a seguir, que será útil para obtermos uma expressão para a razão $\lambda_{k, n}$. 


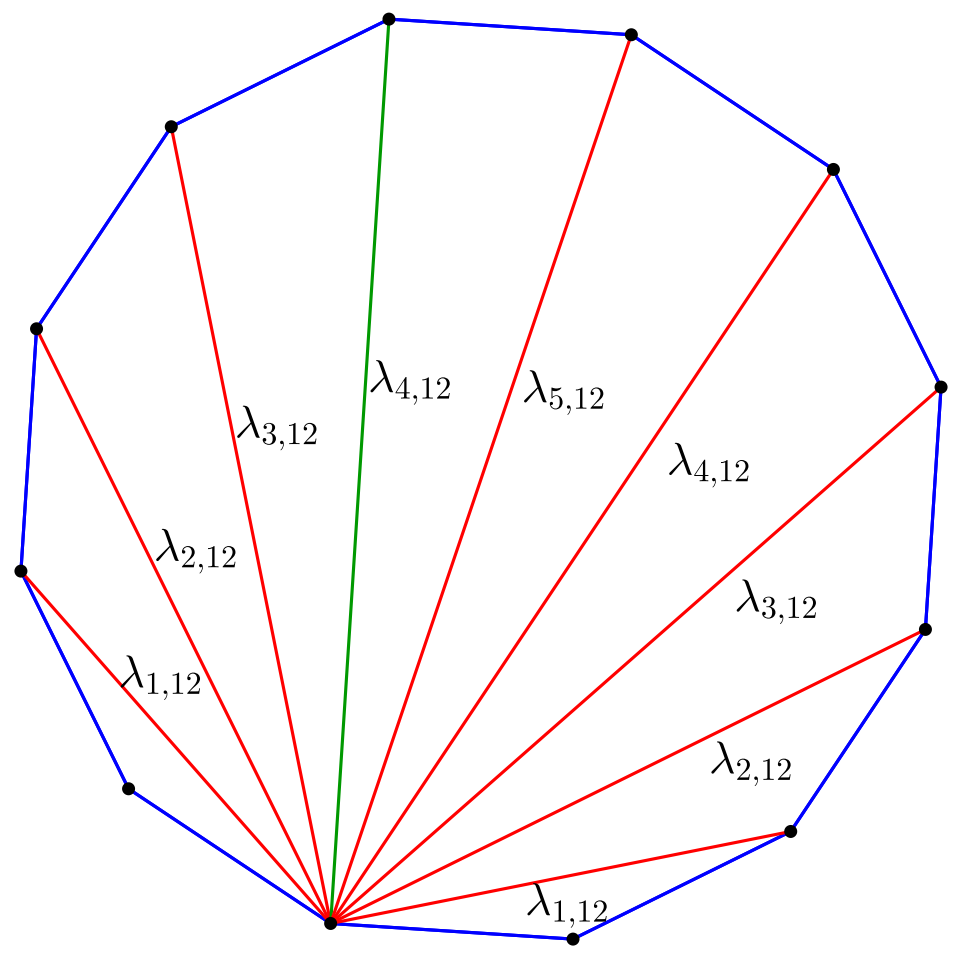

Figura 2: Significado da notação $\lambda_{k, n}$.

Lema 1. Seja $P_{n}$ o polígono regular de $n$ lados inscrito na circunferência unitária $\{z \in \mathbb{C} ;|z|=1\}$ com um vértice no ponto $z=1$. Então a medida do lado é $2 \operatorname{sen}\left(\frac{\pi}{n}\right)$.

Demonstração. A distância entre dois números complexos $z_{1}$ e $z_{2}$ é dada por $\left|z_{2}-z_{1}\right|$. Note que

$$
\begin{aligned}
\left|z_{2}-z_{1}\right|^{2} & =\left(z_{2}-z_{1}\right)\left(\overline{z_{2}-z_{1}}\right) \\
& =z_{2} \overline{z_{2}}-z_{2} \overline{z_{1}}-z_{1} \overline{z_{2}}+z_{1} \overline{z_{1}} \\
& =\left|z_{1}\right|^{2}-\left(z_{1} \overline{z_{2}}+\overline{z_{1} \overline{z_{2}}}\right)+\left|z_{2}\right|^{2} \\
& =\left|z_{1}\right|^{2}+\left|z_{2}\right|^{2}-2 \mathcal{R}\left(z_{1} \overline{z_{2}}\right)
\end{aligned}
$$

Em virtude da equação (3), a medida do lado do polígono regular $P_{n}$ pode ser obtida por $\left|\xi_{1}-\xi_{0}\right|$, onde $\xi_{0}=1$ e $\xi_{1}=\cos \left(\frac{2 \pi}{n}\right)+i \operatorname{sen}\left(\frac{2 \pi}{n}\right)$. Note que

$$
\begin{aligned}
\left|\xi_{1}-\xi_{0}\right|^{2} & =\left|\xi_{0}\right|^{2}+\left|\xi_{1}\right|^{2}-2 \mathcal{R}\left(\xi_{0} \overline{\xi_{1}}\right) \\
& =|1|^{2}+\left|\cos \left(\frac{2 \pi}{n}\right)+i \operatorname{sen}\left(\frac{2 \pi}{n}\right)\right|^{2}-2 \mathcal{R}\left(1 \cdot \overline{\cos \left(\frac{2 \pi}{n}\right)+i \operatorname{sen}\left(\frac{2 \pi}{n}\right)}\right) \\
& =2-2 \cos \frac{2 \pi}{n} \\
& =4 \operatorname{sen}^{2}\left(\frac{\pi}{n}\right), \text { pois } 1-\cos 2 \theta=2 \operatorname{sen}^{2} \theta .
\end{aligned}
$$




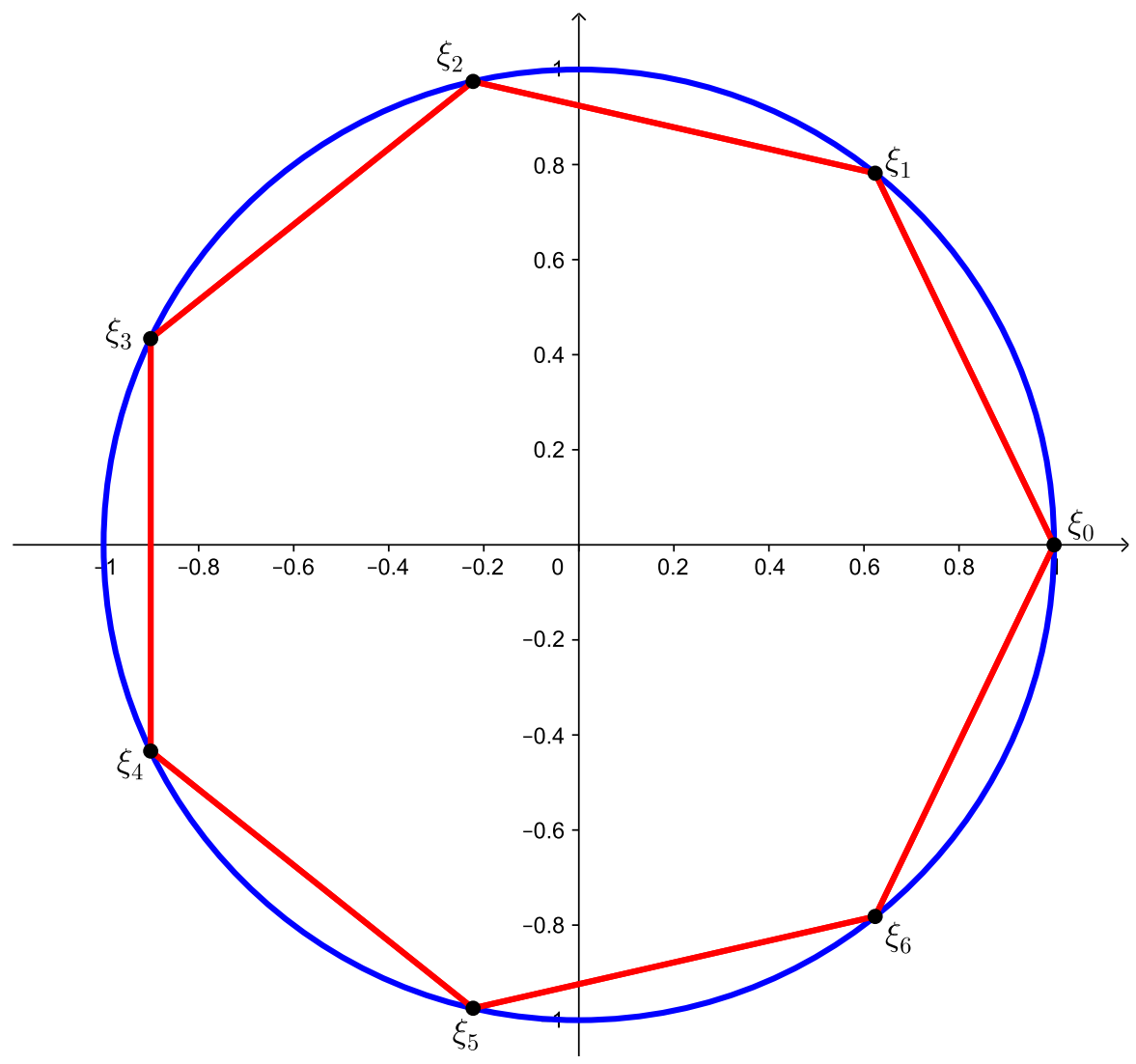

Figura 3: Representação do heptágono regular inscrito no círculo de centro na origem, raio 1 e com um vértice no ponto $(1,0)$.

Portanto, a medida do lado do polígono $P_{n}$ é $2 \operatorname{sen}\left(\frac{\pi}{n}\right)$.

Observe que medida da $k$-ésima diagonal mais curta do polígono $P_{n}$ é dada pela distância entre os vértices $\xi_{0}$ e $\xi_{k+1}$. Ou seja, a medida da $k$-ésima diagonal mais curta é o número $\left|\xi_{k+1}-\xi_{0}\right|$. Utilizando o mesmo raciocínio empregado na demonstração do Lema 1, pode-se concluir que

$$
\left|\xi_{k+1}-\xi_{0}\right|=2 \operatorname{sen} \frac{(k+1) \pi}{n} .
$$

Segue da Equação (4) e do Lema 1 que a razão $\lambda_{k, n}$ entre a medida da $k$-ésima diagonal mais curta do polígono regular de $n$ lados e a medida do seu lado é expressa por

$$
\lambda_{k, n}=\frac{\left|\xi_{k+1}-\xi_{0}\right|}{\left|\xi_{1}-\xi_{0}\right|}=\frac{\operatorname{sen} \frac{(k+1) \pi}{n}}{\operatorname{sen} \frac{\pi}{n}} .
$$

Repare que estudar a questão da incomensurabilidade entre a $k$-ésima diagonal mais curta e o lado de um polígono regular de $n$ lados reduz-se a decidir pela racionalidade ou irracionalidade do 
número $\lambda_{k, n}$. No caso particular da diagonal mais curta temos:

$$
\lambda_{1, n}=\frac{\operatorname{sen} \frac{2 \pi}{n}}{\operatorname{sen} \frac{\pi}{n}}=\frac{2 \operatorname{sen} \frac{\pi}{n} \cos \frac{\pi}{n}}{\operatorname{sen} \frac{\pi}{n}}=2 \cos \frac{\pi}{n} .
$$

Note que $\lambda_{1,4}=\sqrt{2}, \lambda_{1,5}=\phi$ e $\lambda_{1,6}=\sqrt{3}$ que corresponde à razão entre a medida da diagonal mais curta e o lado de um quadrado, pentágono regular e hexágono regular, respectivamente.

\section{Inteiros algébricos e números irracionais}

Nesta seção vamos introduzir o conceito de números inteiros algébricos e apresentar um resultado que será útil no estabelecimento da comensurabilidade ou não entre medida de uma diagonal e a medida do lado de um polígono regular.

Um número (complexo) é dito um inteiro algébrico quando é raiz de uma equação polinomial da forma

$$
x^{r}+c_{r-1} x^{r-1}+\cdots+c_{1} x+c_{0}=0,
$$

onde $r$ é um inteiro positivo e os coeficientes $c_{0}, c_{1}, \ldots, c_{r-1}$ são números inteiros.

Em particular, todo número inteiro $m$ é um inteiro algébrico pois é raiz da equação polinomial $x-m=0$ de coeficientes inteiros. O número $\sqrt{2}$ é um inteiro algébrico pois é uma solução da equação $x^{2}-2=0$.

Proposição 1. Todo inteiro algébrico (real) é inteiro ou irracional.

Demonstração. Suponha que existam inteiros $p$ e $q>0$, primos entre si, tais que o número $\frac{p}{q}$ seja uma solução da equação

$$
x^{r}+c_{r-1} x^{r-1}+c_{r-2} x^{r-2}+\cdots+c_{2} x^{2}+c_{1} x+c_{0}=0,
$$

em que $r$ é um inteiro positivo e os coeficientes $c_{r-1}, c_{r-2}, \ldots, c_{2}, c_{2}$ e $c_{0}$ são números inteiros. Substituindo $x$ por $\frac{p}{q}$ obtemos:

$$
\left(\frac{p}{q}\right)^{r}+c_{r-1}\left(\frac{p}{q}\right)^{r-1}+c_{r-2}\left(\frac{p}{q}\right)^{r-2}+\cdots+c_{2}\left(\frac{p}{q}\right)^{2}+c_{1} \frac{p}{q}+c_{0}=0
$$

Multiplicando ambos os membros da igualdade anterior por $q^{r}$ temos:

$$
p^{r}+c_{r-1} p^{n-1} q+\cdots+c_{1} p q^{r-1}+c_{0} q^{r}=0 .
$$

A equação anterior pode ser escrita como $p^{r}=-q\left(c_{r-1} p^{r-1}+\cdots+c_{1} p q^{r-2}+c_{0} q^{n-1}\right)$. Ou seja,

$$
p^{r}=-q K \text {, onde } K=c_{r-1} p^{r-1}+\cdots+c_{1} p q^{r-2}+c_{0} q^{n-1} .
$$

Se $q>1$, segue da Equação (6) e do Teorema Fundamental da Aritmética que todo fator primo de $q$ é também fator primo de $p$. Mas isso não pode ocorrer, pois $p$ e $q$ são primos entre si. Assim, concluímos que $q=1$. 


\section{O número $\lambda_{n, k}$ é um inteiro algébrico}

Vamos definir, para qualquer $x$ real, o piso ou a parte inteira $\lfloor x\rfloor$ como o único inteiro $r$ tal que $r \leq x<r+1$. Ou seja, $\lfloor x\rfloor$ denota o maior inteiro menor do que ou igual a $x$.

A proposição a seguir mostra que a razão $\lambda_{k, n}$, com $k \geq 0$ e $n \geq 4$ inteiros, pode ser expressa em termos de um polinômio em $2 \cos \frac{\pi}{n}$. Este resultado nos dará a ferramenta necessária para averiguar a irracionalidade de $\lambda_{k, n}$.

Proposição 2. Seja $k$ um inteiro não negativo e $n \geq 4$ um inteiro. Então

$$
\lambda_{k, n}=\frac{\operatorname{sen} \frac{(k+1) \pi}{n}}{\operatorname{sen} \frac{\pi}{n}}=\sum_{j=0}^{\left\lfloor\frac{k}{2}\right\rfloor}(-1)^{j}\left(\begin{array}{c}
k-j \\
j
\end{array}\right)\left(2 \cos \frac{\pi}{n}\right)^{k-2 j} .
$$

Demonstração. A prova será feita por indução em $k$. Se $k=0$ então

$$
\lambda_{0, n}=\frac{\operatorname{sen} \frac{(0+1) \pi}{n}}{\operatorname{sen} \frac{\pi}{n}}=1=\sum_{j=0}^{\left\lfloor\frac{0}{2}\right\rfloor}(-1)^{j}\left(\begin{array}{c}
-j \\
j
\end{array}\right)\left(2 \cos \frac{\pi}{n}\right)^{-2 j} .
$$

(Vamos interpretar o caso em que $k=0$ como o lado o polígono). Se $k=1$ então

$$
\lambda_{1, n}=\frac{\operatorname{sen} \frac{(1+1) \pi}{n}}{\operatorname{sen} \frac{\pi}{n}}=\frac{2 \operatorname{sen} \frac{\pi}{n} \cos \frac{\pi}{n}}{\operatorname{sen} \frac{\pi}{n}}=2 \cos \frac{\pi}{n}=\sum_{j=0}^{\left\lfloor\frac{1}{2}\right\rfloor}(-1)^{j}\left(\begin{array}{c}
1-j \\
j
\end{array}\right)\left(2 \cos \frac{\pi}{n}\right)^{1-2 j} .
$$

Tome agora $k \geq 2$ e vamos admitir que a equação (7) é válida para qualquer inteiro menor do que $k$. Note que

$$
\operatorname{sen} \frac{(k+1) \pi}{n}=\operatorname{sen} \frac{k \pi}{n} \cos \frac{\pi}{n}+\cos \frac{k \pi}{n} \operatorname{sen} \frac{\pi}{n} .
$$

Dividindo a igualdade anterior por sen $\frac{\pi}{n}$, obtemos:

$$
\lambda_{k, n}=\frac{\operatorname{sen} \frac{(k+1) \pi}{n}}{\operatorname{sen} \frac{\pi}{n}}=\frac{\operatorname{sen} \frac{k \pi}{n}}{\operatorname{sen} \frac{\pi}{n}} \cos \frac{\pi}{n}+\cos \frac{k \pi}{n}=\lambda_{k-1, n} \cos \frac{\pi}{n}+\cos \frac{k \pi}{n} .
$$

Por outro lado,

$$
\begin{aligned}
\operatorname{sen} \frac{(k+1) \pi}{n} & =\operatorname{sen} \frac{k \pi}{n} \cos \frac{\pi}{n}+\cos \frac{k \pi}{n} \operatorname{sen} \frac{\pi}{n} \\
& =\operatorname{sen} \frac{k \pi}{n} \cos \frac{\pi}{n}-\cos \frac{k \pi}{n} \operatorname{sen} \frac{\pi}{n}+2 \cos \frac{k \pi}{n} \operatorname{sen} \frac{\pi}{n} \\
& =\operatorname{sen}\left(\frac{k \pi}{n}-\frac{\pi}{n}\right)+2 \cos \frac{k \pi}{n} \operatorname{sen} \frac{\pi}{n} \\
& =\operatorname{sen} \frac{(k-1) \pi}{n}+2 \cos \frac{k \pi}{n} \operatorname{sen} \frac{\pi}{n}
\end{aligned}
$$


Da expressão anterior, concluímos que

$$
\lambda_{k, n}=\frac{\operatorname{sen} \frac{(k+1) \pi}{n}}{\operatorname{sen} \frac{\pi}{n}}=\frac{\operatorname{sen} \frac{(k-1) \pi}{n}}{\operatorname{sen} \frac{\pi}{n}}+2 \cos \frac{k \pi}{n}=\lambda_{k-2, n}+2 \cos \frac{k \pi}{n} .
$$

Por fim, utilizando as equações (8) e (9), respectivamente, temos:

$$
\begin{aligned}
\lambda_{k, n} & =2 \lambda_{k, n}-\lambda_{k, n} \\
& =2\left[\lambda_{k-1, n} \cos \frac{\pi}{n}+\cos \frac{k \pi}{n}\right]-\left[\lambda_{k-2, n}+2 \cos \frac{k \pi}{n}\right] \\
& =2 \lambda_{k-1, n} \cos \frac{\pi}{n}-\lambda_{k-2, n}
\end{aligned}
$$

A partir desse momento, com o propósito de simplificar a notação, vamos escrever $x$ no lugar de $2 \cos \frac{\pi}{n}$. Assim,

$$
\lambda_{k, n}=x \lambda_{k-1, n}-\lambda_{k-2, n} .
$$

Vamos supor, para fixar as ideias, que $k$ é um inteiro ímpar. Portanto, $k \geq 3$. Nesse caso,

$$
\left\lfloor\frac{k-1}{2}\right\rfloor=\frac{k-1}{2} \text { e }\left\lfloor\frac{k-2}{2}\right\rfloor=\frac{k-3}{2} .
$$

Dessa forma, usando a hipótese de indução, temos

$$
\begin{aligned}
& \lambda_{k, n}=x \lambda_{k-1, n}-\lambda_{k-2, n} \\
& =x \sum_{j=0}^{\left\lfloor\frac{k-1}{2}\right\rfloor}(-1)^{j}\left(\begin{array}{c}
k-1-j \\
j
\end{array}\right) x^{k-1-2 j}-\sum_{j=0}^{\left\lfloor\frac{k-2}{2}\right\rfloor}(-1)^{j}\left(\begin{array}{c}
k-2-j \\
j
\end{array}\right) x^{k-2-2 j} \\
& =\sum_{j=0}^{\frac{k-1}{2}}(-1)^{j}\left(\begin{array}{c}
k-1-j \\
j
\end{array}\right) x^{k-2 j}-\sum_{j=0}^{\frac{k-3}{2}}(-1)^{j}\left(\begin{array}{c}
k-2-j \\
j
\end{array}\right) x^{k-2-2 j} \\
& =x^{k}+\sum_{j=1}^{\frac{k-1}{2}}(-1)^{j}\left(\begin{array}{c}
k-1-j \\
j
\end{array}\right) x^{k-2 j}-\sum_{j=1}^{\frac{k-1}{2}}(-1)^{j-1}\left(\begin{array}{c}
k-1-j \\
j-1
\end{array}\right) x^{k-2 j} \\
& =x^{k}+\sum_{j=1}^{\frac{k-1}{2}}(-1)^{j}\left(\begin{array}{c}
k-1-j \\
j
\end{array}\right) x^{k-2 j}+\sum_{j=1}^{\frac{k-1}{2}}(-1)^{j}\left(\begin{array}{c}
k-1-j \\
j-1
\end{array}\right) x^{k-2 j} \\
& =x^{k}+\sum_{j=1}^{\frac{k-1}{2}}(-1)^{j}\left[\left(\begin{array}{c}
k-1-j \\
j
\end{array}\right)+\left(\begin{array}{c}
k-1-j \\
j-1
\end{array}\right)\right] x^{k-2 j} \\
& =x^{k}+\sum_{j=1}^{\frac{k-1}{2}}(-1)^{j}\left(\begin{array}{c}
k-j \\
j
\end{array}\right) x^{k-2 j} \\
& =\sum_{j=0}^{\left\lfloor\frac{k}{2}\right\rfloor}(-1)^{j}\left(\begin{array}{c}
k-j \\
j
\end{array}\right) x^{k-2 j}
\end{aligned}
$$

onde usamos, na penúltima igualdade da cadeia de equações acima, a relação de Stifel e, na última igualdade, o fato que $\frac{k-1}{2}=\left\lfloor\frac{k}{2}\right\rfloor$ quando $k$ é ímpar. A demonstração no caso em que $k$ é par é análoga. 
Se $\alpha$ e $\beta \neq 0$ são dois inteiros algébricos, então os números $\alpha+\beta, \alpha-\beta$ e $\alpha \beta$ são também inteiros algébricos $^{1}$. A prova desse fato encontra-se em [4]. Além disso, se $n$ é um inteiro positivo então $\alpha^{n}$ é um inteiro algébrico. Daí segue que se $\gamma$ é um inteiro algébrico e $f$ é uma função polinomial com coeficientes inteiros, então o número $f(\gamma)$ é também um inteiro algébrico.

Vamos mostrar agora que o número $2 \cos \frac{\pi}{n}$ (com $n$ inteiro positivo) é um inteiro algébrico. Com efeito,

$$
2 \cos \frac{\pi}{n}=\left(\cos \frac{\pi}{n}+i \operatorname{sen} \frac{\pi}{n}\right)+\left(\cos \frac{\pi}{n}-i \operatorname{sen} \frac{\pi}{n}\right)
$$

e os números $\cos \frac{\pi}{n}+i \operatorname{sen} \frac{\pi}{n}$ e $\cos \frac{\pi}{n}-i \operatorname{sen} \frac{\pi}{n}$ são soluções da equação $x^{2 n}-1=0$. De fato, segue da fórmula de De Moivre ${ }^{2}$ que

$$
\begin{aligned}
& \left(\cos \frac{\pi}{n}+i \operatorname{sen} \frac{\pi}{n}\right)^{2 n}-1=\cos (2 \pi)+i \operatorname{sen}(2 \pi)-1=0 \mathrm{e} \\
& \left(\cos \frac{\pi}{n}-i \operatorname{sen} \frac{\pi}{n}\right)^{2 n}-1=\cos (2 \pi)-i \operatorname{sen}(2 \pi)-1=0
\end{aligned}
$$

Em outras palavras, o número $2 \cos \frac{\pi}{n}$ é algébrico pois é a soma de dois inteiros algébricos. Segue da equação (7) que o número $\lambda_{k, n}$ é um inteiro algébrico pois pode ser expresso como um polinômio em $2 \cos \frac{\pi}{n}$ com coeficientes inteiros. Segue da Proposição 1 que a razão $\lambda_{k, n}$ é um número inteiro ou irracional. Nossa conclusão é destacada na proposição a seguir.

Proposição 3. Sejam $n \geq 4$ e $k \geq 1$ números inteiros. O número $\lambda_{k, n}$ é um inteiro algébrico. Ou seja, dado um polígono regular de $n$ lados, a razão entre a medida da k-ésima diagonal mais curta e a medida do seu lado é um número inteiro ou um número irracional.

Portanto, o problema de se decidir pela racionalidade ou irracionalidade do número $\lambda_{k, n}$, com $n \geq 2 k+2$, isto é, decidir pela comensurabilidade ou incomensurabilidade entre o lado e a diagonal do polígono regular, reduz-se a decidir se $\lambda_{k, n}$ é inteiro ou não.

É evidente que se trata de um problema muito mais simples. Para a quarta diagonal mais curta do dodecágono regular, por exemplo, com o auxílio da expressão (5) e de uma calculadora, obtemos o valor $\lambda_{4,12} \approx 3,7321$. Como não é um número inteiro, concluímos que se trata de um número irracional. Em outras palavras, a quarta diagonal mais curta e o lado do dodecágono regular são dois segmentos incomensuráveis. $\mathrm{O}$ mesmo procedimento pode ser realizado com quaisquer outros valores de $k$ e $n$, com $n \geq 2 k+2$.

A tabela a seguir, construída com auxílio da expressão (5), fornece uma aproximação com quatro casas decimais para os números $\lambda_{k, n}$, com $1 \leq k \leq 10$ e $4 \leq n \leq 34$. Note que o único número inteiro na tabela corresponte a razão $\lambda_{2,6}=2$. Assim, no que diz respeito até a décima diagonal

\footnotetext{
${ }^{1} \mathrm{O}$ conjunto de todos os inteiros algébricos forma um subanel de $\mathbb{C}$. No entanto a razão de dois inteiros algébricos não é necessariamente um inteiro algébrico. Por exemplo, segue da Proposição 1 que o número $\frac{1}{2}$ não é um inteiro algébrico, apesar de ser a razão dos inteiros algébricos 1 e 2 .

${ }^{2}$ Dado $z=|z|(\cos \theta+i \operatorname{sen} \theta)$ (onde $\theta$ é um número real) um número complexo não nulo na forma polar e $n$ um número inteiro, então $z^{n}=|z|^{n}(\cos (n \theta)+i \operatorname{sen}(n \theta))$.
} 
mais curta de polígonos regulares com até 34 lados, somente a segunda diagonal mais curta e o lado do hexágono regular formam segmentos comensuráveis.

\begin{tabular}{|c|c|c|c|c|c|c|c|c|c|c|}
\hline$n \backslash k$ & 1 & 2 & 3 & 4 & 5 & 6 & 7 & 8 & 9 & 10 \\
\hline 4 & 1,4142 & & & & & & & & & \\
\hline 5 & 1,6180 & & & & & & & & & \\
\hline 6 & 1,7321 & 2,0000 & & & & & & & & \\
\hline 7 & 1,8019 & 2,2470 & & & & & & & & \\
\hline 8 & 1,8478 & 2,4142 & 2,6131 & & & & & & & \\
\hline 9 & 1,8794 & 2,5321 & 2,8794 & & & & & & & \\
\hline 10 & 1,9021 & 2,6180 & 3,0777 & 3,2361 & & & & & & \\
\hline 11 & 1,9190 & 2,6825 & 3,2287 & 3,5133 & & & & & & \\
\hline 12 & 1,9319 & 2,7321 & 3,3461 & 3,7321 & 3,8637 & & & & & \\
\hline 13 & 1,9419 & 2,7709 & 3,4389 & 3,9070 & 4,1481 & & & & & \\
\hline 14 & 1,9499 & 2,8019 & 3,5135 & 4,0489 & 4,3813 & 4,4940 & & & & \\
\hline 15 & 1,9563 & 2,8271 & 3,5743 & 4,1654 & 4,5743 & 4,7834 & & & & \\
\hline 16 & 1,9616 & 2,8478 & 3,6245 & 4,2620 & 4,7357 & 5,0273 & 5,1258 & & & \\
\hline 17 & 1,9659 & 2,8649 & 3,6664 & 4,3430 & 4,8716 & 5,2344 & 5,4190 & & & \\
\hline 18 & 1,9696 & 2,8794 & 3,7017 & 4,4115 & 4,9872 & 5,4115 & 5,6713 & 5,7588 & & \\
\hline 19 & 1,9727 & 2,8916 & 3,7317 & 4,4699 & 5,0862 & 5,5638 & 5,8896 & 6,0548 & & \\
\hline 20 & 1,9754 & 2,9021 & 3,7574 & 4,5201 & 5,1716 & 5,6957 & 6,0796 & 6,3138 & 6,3925 & \\
\hline 21 & 1,9777 & 2,9111 & 3,7796 & 4,5636 & 5,2457 & 5,8106 & 6,2457 & 6,5413 & 6,6907 & \\
\hline 22 & 1,9796 & 2,9190 & 3,7989 & 4,6015 & 5,3104 & 5,9112 & 6,3917 & 6,7420 & 6,9552 & 7,0267 \\
\hline 23 & 1,9814 & 2,9258 & 3,8158 & 4,6347 & 5,3672 & 5,9998 & 6,5206 & 6,9199 & 7,1903 & 7,3268 \\
\hline 24 & 1,9829 & 2,9319 & 3,8306 & 4,6639 & 5,4174 & 6,0781 & 6,6349 & 7,0781 & 7,4002 & 7,5958 \\
\hline 25 & 1,9842 & 2,9372 & 3,8438 & 4,6898 & 5,4618 & 6,1477 & 6,7367 & 7,2194 & 7,5882 & 7,8374 \\
\hline 26 & 1,9854 & 2,9419 & 3,8555 & 4,7128 & 5,5014 & 6,2098 & 6,8277 & 7,3459 & 7,7571 & 8,0552 \\
\hline 27 & 1,9865 & 2,9461 & 3,8659 & 4,7334 & 5,5368 & 6,2654 & 6,9093 & 7,4598 & 7,9093 & 8,2519 \\
\hline 28 & 1,9874 & 2,9499 & 3,8752 & 4,7518 & 5,5686 & 6,3155 & 6,9829 & 7,5624 & 8,0469 & 8,4302 \\
\hline 29 & 1,9883 & 2,9532 & 3,8836 & 4,7684 & 5,5973 & 6,3606 & 7,0493 & 7,6554 & 8,1717 & 8,5922 \\
\hline 30 & 1,9890 & 2,9563 & 3,8912 & 4,7834 & 5,6232 & 6,4014 & 7,1095 & 7,7397 & 8,2851 & 8,7397 \\
\hline 31 & 1,9897 & 2,9591 & 3,8980 & 4,7970 & 5,6467 & 6,4385 & 7,1642 & 7,8164 & 8,3884 & 8,8744 \\
\hline 32 & 1,9904 & 2,9616 & 3,9043 & 4,8093 & 5,6681 & 6,4723 & 7,2141 & 7,8865 & 8,4829 & 8,9976 \\
\hline 33 & 1,9909 & 2,9639 & 3,9099 & 4,8206 & 5,6876 & 6,5031 & 7,2597 & 7,9506 & 8,5694 & 9,1107 \\
\hline 34 & 1,9915 & 2,9659 & 3,9151 & 4,8309 & 5,7054 & 6,5313 & 7,3015 & 8,0093 & 8,6489 & 9,2146 \\
\hline
\end{tabular}

Tabela 1: Razão $\lambda_{n, k}$ entre as medidas da $k$-ésima diagonal mais curta e do lado.

\section{Conclusão}

É importante notar que o problema da comensurabilidade entre a medida do lado e a medida de uma diagonal de um polígono regular não foi completamente respondida. No entanto, a questão foi reduzida em um problema mais simples. De fato, estudar a natureza quanto à racionalidade ou irracionalidade do número $\lambda_{k, n}$ reduziu-se, por meio da Proposição 1 , a decidir se o número é inteiro ou não. Reduzir um problema em outro mais simples é um procedimento muito comum em matemática. Por exemplo, decidir se $\sqrt[3]{120}$ é racional ou irracional, por meio dessa abordagem, 
torna-se simples pois basta notar que tal número é um inteiro algébrico (pois é raiz da equação $\left.x^{3}-120=0\right)$ e por outro lado não é inteiro, já que $4=\sqrt[3]{64}<\sqrt[3]{120}<\sqrt[3]{125}<5$. Assim, da Proposição 1 concluímos que $\sqrt[3]{120}$ é irracional.

Apesar de sermos capazes de decidir se $\lambda_{k, n}$ é ou não irracional, fixado $k$ e $n$, por meio da Proposição 1 e da expressão (5) o problema mais geral, de saber para quais inteiros $k$ e $n$ o número $\lambda_{k, n}$ é irracional, não foi respondido.

O presente artigo apresentou uma interessante conexão entre a álgebra e a geometria. Com efeito, o problema gemétrico da comensurabilidade entre o lado e uma diagonal de um polígono regular, que podemos localizar suas origens na matemática da Grécia Antiga, foi abordado utilizando conceitos de inteiros algébricos, além dos números complexos.

Acreditamos que os assuntos aqui tratados são de interesse para o professor de matemática já que se trata de números irracionais, tópico presente no $9^{\mathrm{o}}$ ano e na $1^{\mathrm{a}}$ série do Ensino Médio, e de segmentos comensuráveis e incomensuráveis que são muitas vezes utilizados como uma introdução aos números irracionais. Tal assunto pode ser retomado na $3^{\text {a }}$ série do Ensino Médio, quando os alunos tomam contato com os números complexos. Trata-se de uma interessante aplicação dos números complexos para se resolver um importante problema de geometria.

\section{Referências}

[1] ATRACTOR. Disponível em: http://www.atractor.pt/mat/incomensurabilidade/

[2] HEFEZ, ABRAMO. VIllelA, MARIA LÚCIA Polinômios e Equações Algébricas, Coleção Profmat, Rio de Janeiro: SBM, 2012.

[3] HAVIL, JULIAN The Irrationals: A Story of the Numbers You Can't Count On. New Jersey: Princeton University Press, 2012.

[4] MARTINEZ, F. B. et al. Teoria dos Números: Um passeio com Primos e outros Números Familiares pelo Mundo Inteiro. Impa, Rio de Janeiro: Impa, 2010.

[5] ROQUE, TATIANA. História da Matemática: uma visão crítica, desfazendo mitos e lendas. Rio de Janeiro: Zahar, 2012.

Ronald S. de M. Pinto

Colégio Pedro II $<$ ronaldsimoes@gmail.com>

Diego Alves

Colégio Pedro II <diegoalv.mat@gmail.com>

Recebido: 21/04/2019

Publicado: 28/06/2019 